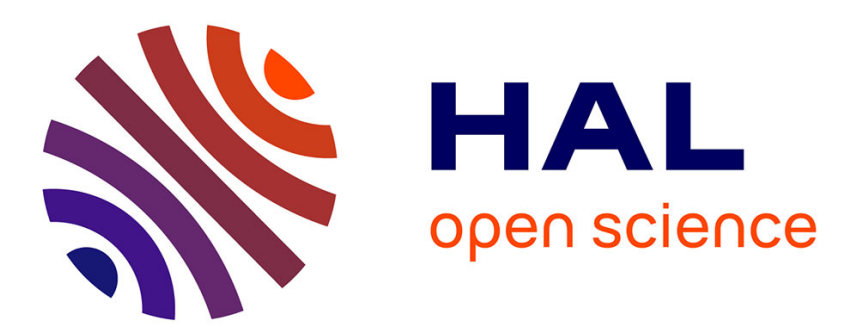

\title{
Effects of bending portions of the air column on the acoustical resonances of a wind instrument
}

Simon Félix, Jean-Pierre Dalmont

\section{To cite this version:}

Simon Félix, Jean-Pierre Dalmont. Effects of bending portions of the air column on the acoustical resonances of a wind instrument. Journal of the Acoustical Society of America, 2012, 131 (5), pp.41644172. 10.1121/1.3699267. hal-00643184v2

\section{HAL Id: hal-00643184 \\ https://hal.science/hal-00643184v2}

Submitted on 29 Mar 2012

HAL is a multi-disciplinary open access archive for the deposit and dissemination of scientific research documents, whether they are published or not. The documents may come from teaching and research institutions in France or abroad, or from public or private research centers.
L'archive ouverte pluridisciplinaire HAL, est destinée au dépôt et à la diffusion de documents scientifiques de niveau recherche, publiés ou non, émanant des établissements d'enseignement et de recherche français ou étrangers, des laboratoires publics ou privés. 
Effects of bending portions of the air column on the acoustical resonances of a wind instrument

\author{
Simon Félix a) and Jean-Pierre Dalmont \\ LAUM, \\ CNRS, \\ Université du Maine, \\ avenue Olivier Messiaen, \\ 72085 Le Mans, \\ France. \\ C. J. Nederveen \\ Acacialaan 20, \\ 2641 AC Pijnacker, \\ Netherlands
}

(Dated: March 29, 2012)

Bends in musical wind instruments 1 


\begin{abstract}
The need to keep long wind musical instruments compact imposes the bending of portions of the air column. Although manufacturers and players mention its effects as being significant, the curvature is generally not included in physical models and only a few studies, in only simplified cases, attempted to evaluate its influence. The aim of the study is to quantify the influence of the curvature both theoretically and experimentally. A multimodal formulation of the wave propagation in bent ducts is used to calculate the resonances frequencies and input impedance of a duct segment with a bent portion. From these quantities an effective length is defined. Its dependence on frequency is such that, compared to an equivalent straight tube, the shift in resonance frequencies in a tube with bent sections is not always positive, as generally stated. The curvature does not always increase the resonances frequencies, but may decrease them, resulting in a complex inharmonicity. An experimental measurement of the effect of the curvature is also shown, with good agreement with theoretical predictions.
\end{abstract}

PACS numbers: 43.75.Ef, 43.75.Fg 43.20.Mv, 


\section{INTRODUCTION}

Long wind musical instruments are often made with bends to keep them compact. Although it is generally stated, following Rayleigh's argument, that "straightness is not a matter of importance,, 1$]$ bending portions of the air column may affect the tuning in a musically significant way. Several authors attempted to quantify the effect of the curvature on the tuning. Nederveen ${ }^{[23}$ and later Keefe and Benade ${ }^{\sqrt{4}}$ showed that in a bent duct, in the long wavelength limit, the inertance is lowered relative to its value in a straight duct having the same cross-section. The curvature induces an increase in resonance frequencies. In other words, the length correction due to the curvature in a duct is negative. Experimental studies reported in the litterature ${ }^{3 / 5}$ confirm this qualitative result at low frequency. Following these studies, methods for compensating bends in air columns when designing wind instruments have been proposed, as, for example, a reduction of the bore diameter at bends, combined with a lengthening when the frequency is to be the same as the straight duct of the original length.

However, reducing the bore diameter at bends to compensate for the tuning change supposes that this change is weakly frequency-dependent, or, at least, that the frequency shift, or the length correction, remains the same sign over the musically useful frequency range.

It is shown in this paper that the frequency shift in a bend may vary significantly over frequency and that its sign changes. The curvature does not always increase the resonance frequencies, but may decrease them, resulting in a complex inharmonicity. Two ways of characterizing the effect of the curvature are described in the following: the direct computation of the resonance frequencies in a duct with a bend, and the computation of its input impedance. Both quantities are calculated using several theoretical or numerical methods

\footnotetext{
a)Electronic address: simon.felix@univ-lemans.fr
} 
that are first described to detail all the necessary quantities and notations (Sec. II). Then results are given and discussed (Sec. III), followed by an experimental verification (Sec. IV).

\section{METHODS}

\section{A. General formulation}

For simplicity and in order to clearly point out the effect of bending, the air column is supposed to have a constant, circular, cross-section. Since any such waveguide can be represented by a succession of cylindrical and toroidal segments, we will consider in the following the duct drawn in Fig. 1. The curvature of the middle segment is quantified by the adimensional axis curvature $\kappa=a / R_{0}$, where $R_{0}$ is the axis radius of curvature.

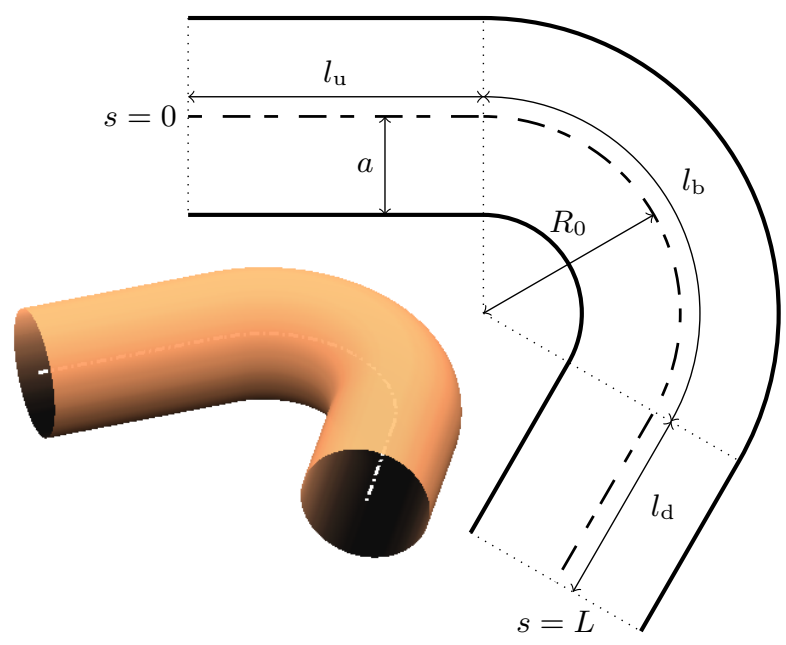

FIG. 1. Duct with a bent portion. $s$ is the abscissa along the waveguide axis, measured from the input of the duct (top), and $L=l_{\mathrm{u}}+l_{\mathrm{b}}+l_{\mathrm{d}}$ is the total length of the duct axis.

The waveguide is filled with an inviscid and homogeneous medium, with density $\rho_{0}$ and sound velocity $c_{0}$ (in Sec. IV, approximations of the viscothermal losses are introduced to allow the comparison with experimental results). Under the assumption of linear propagation, the acoustic pressure and axial velocity in the waveguide are the solutions of the coupled 
equations [the time dependence $\exp (-\mathrm{j} \omega t)$ is omitted]

$$
\frac{\partial}{\partial s}\left(\begin{array}{c}
p \\
A v_{s}
\end{array}\right)=\left(\begin{array}{cc}
0 & \mathrm{j} k Z_{\mathrm{c}} h_{s} \\
-\frac{h_{s}}{\mathrm{j} k Z_{\mathrm{c}}}\left(\Delta_{\perp}^{(\kappa)}+k^{2}\right) & 0
\end{array}\right)\left(\begin{array}{c}
p \\
A v_{s}
\end{array}\right)
$$

where $A=\pi a^{2}$ is the cross-section area, $Z_{\mathrm{c}}=\rho_{0} c_{0} / A$ is the characteristic impedance, and $k=\omega / c_{0}$ is the wavenumber. In the bent segment, $\Delta_{\perp}^{(\kappa)}$ is the transverse Laplacian in the toroidal coordinates $(s, r, \theta)$, with $s$ the abscissa along the waveguide axis and $(r, \theta)$ the polar coordinates in the transverse cross-section: 78

$$
\Delta_{\perp}^{(\kappa)}=\frac{1}{r h_{s}}\left[\frac{\partial}{\partial r}\left(r h_{s} \frac{\partial}{\partial r}\right)+\frac{\partial}{\partial \theta}\left(\frac{h_{s}}{r} \frac{\partial}{\partial \theta}\right)\right]
$$

with $h_{s}=1-\kappa r \cos \theta / a$. In the cylindrical segments, $\kappa=0$ and $\Delta_{\perp}^{(0)}$ is the transverse Laplacian in cylindrical coordinates:

$$
\Delta_{\perp}^{(0)}=\frac{1}{r} \frac{\partial}{\partial r}\left(r \frac{\partial}{\partial r}\right)+\frac{1}{r^{2}} \frac{\partial^{2}}{\partial \theta^{2}} .
$$

The resonances of the duct combination can be studied in two ways:

1. by a direct calculation of the resonance frequencies,

2. or by calculating the impedance at the input of the duct.

\section{Resonance frequencies}

The general principle for calculating the resonance frequencies is the following. From the coupled equations (1), one deduces a relation between the acoustic fields at each end of the duct. For example, the pressure and axial velocity field at the output $(s=L)$ are written as function of the fields at the input $(s=0)$ :

$$
\left(p(L, r, \theta), v_{s}(L, r, \theta)\right)=H\left(p(0, r, \theta), v_{s}(0, r, \theta)\right)
$$

with $H$ an operator and frequency dependent. Then, given this relation and the boundary conditions at each end of the duct, a dispersion relation is written, the solutions of which 
are the resonance frequencies $f_{i}^{(\kappa)}(i=1,2,3, \ldots)$ in the duct combination. Practically, this problem is solved by discretization of the solutions (cf. §II.C) or the differential operators (see $\S$ II.D) in Eq. (1), so that Eq. (4) and the dispersion relation become linear matrix equations.

In the following, the curvature induced shift of the resonance frequencies will be expressed as a correction to the length $L$ of the duct:

$$
\Delta L_{i}=\left(\frac{f_{i}^{(0)}}{f_{i}^{(\kappa)}}-1\right) L
$$

where the $f_{i}^{(0)}$ are the resonance frequencies in a cylindrical duct having the same radius $a$ and length $L$ as the duct, with the same boundary conditions imposed at the ends. For simple end conditions, the $f_{i}^{(0)}$ are known analytically. For example, if one imposes at the input end $(s=0)$ a Neumann condition $\partial p / \partial s=0$ and, at the output end $(s=L)$, a Dirichlet condition $p=0$ as the simplest, non radiating, open end condition, then the resonance frequencies of this "closed-open" duct are

$$
f_{i}^{(0)}=\left(i-\frac{1}{2}\right) \frac{c_{0}}{2 L}, \quad i=1,2,3, \ldots
$$

for frequencies under the cutoff of the first superior mode in the duct, that is, $f_{\mathrm{c}}=\gamma_{10} c_{0} / 2 \pi a$, with $\gamma_{10} \simeq 1.8412$ the first positive zero of the first derivative of the Bessel function of the first kind $J_{1}$.

\section{Input impedance}

Classically, the resonator composing of a wind instrument is characterized by calculating or measuring its input impedance

$$
Z_{\text {in }}^{(\kappa)}=\bar{p}(0) / A \bar{v}_{s}(0)
$$

as a function of the frequency, where $\bar{p}(0)$ and $A \bar{v}_{s}(0)$ are, respectively, the mean pressure and volume velocity at the input end of the duct. The impedance proves valuable to study 
the linear acoustic properties of the instrument, since, in particular, its peaks are strongly linked to the playing frequencies of the instrument.

In a cylindrical duct, assuming that only plane waves can propagate $\left(f<f_{\mathrm{c}}\right)$, the input impedance reads as

$$
Z_{\text {in }}^{(0)}=Z_{\mathrm{c}} \tanh \left(-\mathrm{j} k L+\operatorname{arctanh}\left[\frac{Z(L)}{Z_{\mathrm{c}}}\right]\right)
$$

with $Z(L)$ the value of the impedance at the output end of the duct. If the curvature of some region of the duct is not zero, then the input impedance cannot be written analytically. Therefore, approximate analytical solutions (cf. Sec. II.B) or more complex formulations (cf. Sec. II.C) are used.

Once the input impedance $Z_{\text {in }}$ is computed, it can be recalculated into a length correction. Using Eq. (8), an effective length of the duct is defined:

$$
L_{\text {eff. }}=\operatorname{Re}\left[-\frac{1}{\mathrm{j} k}\left(\operatorname{arctanh}\left[\frac{Z_{\text {in }}^{(\kappa)}}{Z_{\mathrm{c}}}\right]-\operatorname{arctanh}\left[\frac{Z(L)}{Z_{\mathrm{c}}}\right]\right)\right],
$$

leading to the length correction

$$
\Delta L(f)=L_{\text {eff. }}-L
$$

Following are explained three methods to solve the general equations given above and characterize the effect of the curvature on the resonances of the duct.

\section{B. Low frequency approximation}

Considering the problem in the long wavelength limit, Nederveen ${ }^{213}$ assumes the pressure

field in a bend to be constant over the circular cross-section: $p(s, r, \theta)=\bar{p}(s)$. Then, Eq. (1) can be simplified by removing the term involving $\Delta_{\perp}^{(\kappa)} p$, and the integration of the equation over the cross-section leads to

$$
\frac{\partial}{\partial s}\left(\begin{array}{c}
\bar{p} \\
A \bar{v}_{s}
\end{array}\right)=\left(\begin{array}{cc}
0 & \mathrm{j} k \alpha(\kappa) Z_{\mathrm{c}} \\
\mathrm{j} k / Z_{\mathrm{c}} & 0
\end{array}\right)\left(\begin{array}{c}
\bar{p} \\
A \bar{v}_{s}
\end{array}\right),
$$


where

$$
\alpha=\frac{\frac{1}{2} \kappa^{2}}{1-\sqrt{1-\kappa^{2}}}<1 .
$$

Then, the effect of bending a duct is shown as a perturbation to the inertance, lowered relative to its value in a straight duct. By decomposing the toroidal waveguide into a set

of parallel rectangular slices, Keefe and Benade ${ }^{4}$ propose in 1983 another expression of the inertance correction, that is shown to be analytically identical the Nederveen's formula. ${ }^{3}$

This low frequency approximation of the evolution equations for $p$ and $v_{s}$ is amenable to an analytical solution for the input impedance ${ }^{3}$ and, as it will be shown later in the paper, it gives a good approximation of the propagation constant of the first bend mode in the long wavelength limit. However, the assumption of a uniform pressure over the cross-section is a strong assumption, since, even below the cutoff frequency $f_{\mathrm{c}}$, the variations of the pressure and velocity over the cross-section are not negligible. ${ }^{79}$. Therefore, this solution is limited to a very low frequency range and cannot account for the dependence on frequency of the effect of curvature.

To take into account the transverse variations of the pressure and velocity fields, higher order modes, even though they are evanescent, must be taken into account. This is the aim of the following method.

\section{Multimodal method}

The wave field in a straight duct is commonly written as a series decomposition on the basis of the transverse modes

$$
\phi_{n}^{(0)}(r, \theta)=\Lambda_{n} J_{\mu}\left(\gamma_{\mu \nu} \frac{r}{a}\right) \sin \left(\mu \theta+\frac{\sigma \pi}{2}\right)
$$

where $\Lambda_{n}$ is a normalizing factor and $\gamma_{\mu \nu}$ is the $(\nu+1)$ th zero of $J_{\mu}^{\prime}$, the derivative of the ith-order Bessel function of the first kind [the modes are sorted in increasing order of their cutoff frequency, such that $\mathrm{n}$ is a integer index, referring to the triplet $(\mu, \nu, \sigma)$ of the usual circumferential, radial, and symmetry indexes. ${ }^{7 / 8} \sigma$ is either 0 or $1 . n=0$ denotes the plane wave mode]. These modes are the well known eigenfunctions of the transverse Laplacian 
$\Delta_{\perp}^{(0)}$ in the two-dimensional (2D) circular cross-section of the waveguide. In a bend, the transverse Laplacian $\Delta_{\perp}^{(\kappa)}$ is not separable in toroidal coordinates; therefore the bend modes $\phi_{n}^{(\kappa)}$ cannot be calculated analytically. However, since the basis $\left\{\phi_{n}^{(0)}\right\}_{n \geq 0}$ is a complete orthogonal basis of the circular cross-section, it can be used as a local transverse basis to exactly decompose the wave field:

$$
\begin{aligned}
& p(s, r, \theta)=\sum_{n \geq 0} \phi_{n}^{(0)}(r, s) P_{n}(s), \\
& A v_{s}(s, r, \theta)=\sum_{n \geq 0} \phi_{n}^{(0)}(r, s) U_{n}(s) .
\end{aligned}
$$

The projection of Eq. 11 on the basis $\left\{\phi_{n}^{(0)}\right\}$ gives the matrix coupled equations for the vectors $\vec{P}$ and $\vec{U}$ of the components $P_{n}$ of pressure and $U_{n}$ of volume velocity:

$$
\frac{\partial}{\partial s}\left(\begin{array}{l}
\vec{P} \\
\vec{U}
\end{array}\right)=\left(\begin{array}{cr}
0 & \mathrm{j} k Z_{\mathrm{c}}(\mathcal{I}-\kappa \mathcal{A}) \\
\frac{1}{\mathrm{j} k Z_{\mathrm{c}}}\left[\mathcal{H}^{2}(\mathcal{I}-\kappa \mathcal{A})+\frac{\kappa}{a^{2}} \mathcal{B}\right] & 0
\end{array}\right)\left(\begin{array}{l}
\vec{P} \\
\vec{U}
\end{array}\right),
$$

where $\mathcal{I}$ is the identity matrix, $\mathcal{H}$ is a diagonal matrix given by $\mathcal{H}_{n n}^{(0)}=\mathrm{j} k_{n}^{(0)}, k_{n}^{(0)}=$ $\sqrt{k^{2}-\left(\gamma_{\mu \nu} / a\right)^{2}}$ the propagation constant of the mode $\phi_{n}^{(0)}$, and $\mathcal{A}$ and $\mathcal{B}$ are constant matrices, functions of $\left\{\phi_{n}^{(0)}\right\}$.

This formulation calls for several comments:

- Taking only one mode into account in Eq. (16) does not give Eq. (11). This is because projecting $p$ and $A v_{s}$ on the plane wave mode $\phi_{0}^{(0)}$ only is equivalent to assuming that both the pressure and axial velocity are uniform over the cross-section. Logically, no effect of the curvature arises at this order of the modal decomposition.

- Since the matrix coefficients in the system (16) are constant along the axis, the solutions for the wavefield, the scattering matrix or the impedance matrix (see below) can be obtained algebraically, as functions of the matrix ${ }^{7 / 110}$

$$
\mathcal{M}=(\mathcal{I}-\kappa \mathcal{A})\left[\mathcal{H}^{2}(1-\kappa \mathcal{A})+\frac{\kappa}{a^{2}} \mathcal{B}\right]
$$


- In the case of a straight waveguide segment $(\kappa=0)$, the matrix $\mathcal{M}$ reduces to $\mathcal{H}^{2}$, then the solution of Eq. (16) is the classical modal decomposition mentioned above.

- The bend modes $\phi_{n}^{(\kappa)}$ appear as the basis that diagonalizes the matrix $\mathcal{M}$ when an infinite number of modes is taken into account $\frac{9111}{}$ It is thus possible to approximate these modes and their propagation constants $k_{n}^{(\kappa)}$ by computing the eigensolutions of the truncated matrix $\mathcal{M}$.

\section{Resonance frequencies}

In order to derive a dispersion relation and calculate the resonance frequencies in the duct, the vector field $\vec{P}$ at the left and right ends of the segment is split into ingoing waves $\vec{A}$ and outgoing waves $\vec{B}$. The scattering matrix relates outgoing waves to ingoing waves:

$$
\left(\begin{array}{c}
\vec{B}_{\text {left }} \\
\vec{B}_{\text {right }}
\end{array}\right)=\mathcal{S}(f)\left(\begin{array}{l}
\vec{A}_{\text {left }} \\
\vec{A}_{\text {right }}
\end{array}\right) .
$$

For the kinds of duct we are interested in (toroidal and cylindrical segments), $\mathcal{S}$ can be algebraically calculated ${ }^{7}$ Since the same basis $\left\{\phi_{n}^{(0)}\right\}$ is used for the modal decomposition in each segment, whatever the value of the curvature, writing the continuity at the interfaces is straightforward. The vector pressure and volume velocity fields $\vec{P}(s)$ and $\vec{U}(s)$ are continuous and, as a consequence, the scattering matrix of a set of connected segments can be explicitly written as function of the scattering matrices of each segment.$^{7}$ Once the scattering matrix of the duct combination is calculated and given the boundary conditions, the dispersion relation is written. For example, for the "closed-open" waveguide, the boundary conditions are $\vec{B}_{\text {left }}=\vec{A}_{\text {left }}$ and $\vec{B}_{\text {right }}=-\vec{A}_{\text {right }}$, then

$$
\operatorname{det}\left[\mathcal{S}(f)-\left(\begin{array}{rr}
\mathcal{I} & 0 \\
0 & -\mathcal{I}
\end{array}\right)\right]=0,
$$

where $\mathcal{I}$ is the identity matrix and det denotes the determinant. The resonance frequencies are the solution of Eq. (19). 
In the case of a straight duct, using 12 modes $\phi_{n}^{(0)}$, the solutions of Eq. 19 differ from the theoretical results (Eq. 6) by less than $10^{-12} \%$, thus mainly limited by the numerical precision of the computer.

\section{Input impedance}

In the frame of the multimodal formalism, an impedance matrix can be defined, fulfilling

$$
\vec{P}(s)=\mathcal{Z}(s) \vec{U}(s)
$$

(throughout this paper, the classical scalar impedance is denoted with a roman capital $Z$, while a script letter is used to denote the impedance matrix, as well as all matrix variables). It is governed by a matrix Riccati equation, ${ }^{9|12| 13}$ a solution of which can be written algebraically in the case of a bent or straight segment as studied in this paper. The impedance matrix is continuous at the interfaces between two segments.

Once the boundary condition at the output end is expressed as a matrix $\mathcal{Z}(L)$, the input impedance matrix $\mathcal{Z}^{(\text {in) }}$ of the duct is calculated. As, by definition, $P_{0}=\bar{p}$ and $U_{0}=A \bar{v}_{s}$, we can write

$$
\bar{p}^{(\mathrm{in})}=\mathcal{Z}_{00}^{(\mathrm{in})} A \bar{v}_{s}^{(\mathrm{in})}+\sum_{n>0} \mathcal{Z}_{0 n}^{(\mathrm{in})} U_{n}
$$

Below the first cut-off and far from discontinuities, sources, or other regions where a mode coupling may occur, $U_{n>0} \rightarrow 0$. Thus, providing that $l_{\mathrm{u}}$ is large enough, the scalar input impedance $z_{\text {in }}$ can be approximated by the top left value $\mathcal{Z}_{00}^{(\text {in) }}$ of the impedance matrix:

$$
Z_{\mathrm{in}}^{(\kappa)}=\frac{\bar{p}^{(\mathrm{in})}}{A \bar{v}_{s}^{(\mathrm{in})}} \simeq \mathcal{Z}_{00}^{(\mathrm{in})}
$$

In the case of a straight duct, using 12 modes $\phi_{n}^{(0)}$ to compute the impedance matrix, its corner value $\mathcal{Z}_{00}^{(\text {in) }}$ differs from the analytical solution 8 by roughly $10^{-12} \%$ over the frequency range of interest (from 0 to the cutoff $f_{\mathrm{c}}$ ), certainly depending on the computer precision. 


\section{Finite difference method}

Resonance frequencies of pipe combinations were independently computed numerically using the finite difference method (FDM). A discretization grid is fitted in the tube combination, as schematically shown in Fig. 2 .

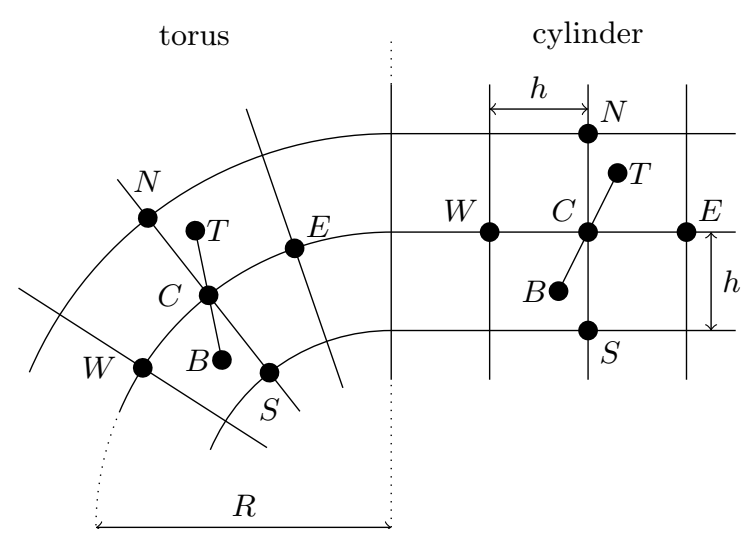

FIG. 2. Grid layout for the FDM solution in a bent segment connected to a straight segment.

The central point $C$ is surrounded by points to the north $(N)$, south $(S)$, west $(W)$, and east $(E)$, and perpendicular to the plane through these points by top $(T)$ and bottom $(B)$

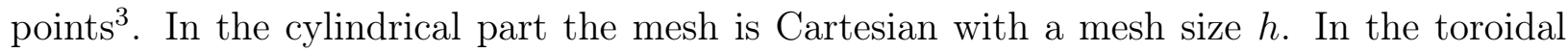
part, $R$ is the local radius of curvature, $R_{0}$ the radius of curvature of the center line at which radius the mesh size is equal to $h$, so the angle between two adjacent grid lines is $h / R_{0}$.

The discretization equation for the Helmholtz equation in the toroidal part in cylindrical coordinates is

$$
\left(1+\frac{h}{2 R}\right) \frac{p_{N}-p_{C}}{h^{2}}+\left(1-\frac{h}{2 R}\right) \frac{p_{S}-p_{C}}{h^{2}}+\left(\frac{R_{0}}{R}\right)^{2} \frac{p_{E}-2 p_{C}+p_{W}}{h^{2}}+\frac{p_{T}-2 p_{C}+p_{B}}{h^{2}}+k^{2} p_{C}=0 .
$$

The conditions in the cylindrical part are found by letting $R$ tend to infinity while $\xi=$ $R / R_{0} \rightarrow 1$. At the connection of the two areas where a torus joins a cylinder, the conditions are more complicated. The terms of the Laplacian are obtained from the flow through the boundary planes of a control volume formed around the central point. ${ }^{[1415]}$ For example, for 
a western torus connected to an eastern cylinder, the first three terms of Eq. (23) change into

$$
\begin{aligned}
& {\left[1+\xi\left(1+\frac{h}{2 R}\right)\right] \frac{p_{N}-p_{C}}{(1+\xi) h^{2}}+\ldots} \\
& {\left[1+\xi\left(1-\frac{h}{2 R}\right)\right] \frac{p_{S}-p_{C}}{(1+\xi) h^{2}}+\ldots} \\
& 2 \frac{p_{E}-p_{C}}{(1+\xi) h^{2}}+\left(\frac{2}{\xi}\right) \frac{p_{W}-p_{C}}{(1+\xi) h^{2}} .
\end{aligned}
$$

Studied were the situations with ideal open end and closed end. In the first case the pressure is set to zero at the pipe end. In the second case the velocity is zero. To find the condition at the boundary consider point $C$ to be located in the boundary on the right-hand side of the cylinder (Fig. 2). The pressure in a point $2 h$ to the west of point $C$ is denoted $p_{2 W}$. For the first and second derivatives in the axial direction $p^{\prime}$ and $p^{\prime \prime}$ we have $e^{16 \mid 17}$

$$
\begin{aligned}
& p^{\prime}=\left(p_{2 W}-4 p_{W}+3 p_{C}\right) / 2 h \\
& p^{\prime \prime}=\left(p_{2 W}-2 p_{W}+p_{C}\right) / h^{2}
\end{aligned}
$$

The condition of zero velocity is $p^{\prime}=0$. Eliminating $p_{2 W}$ from the two equations gives

$$
p^{\prime \prime}=\left(2 p_{W}-2 p_{C}\right) / h^{2}
$$

This result can also be obtained by imagining a virtual point $E$ which acts as a mirror of point $W$ in which the pressure is the same. More elaborate expressions are possible, $\frac{16 \mid 17}{1}$ they were tried, but were not found to give better results. Similar expressions are used at the side walls, where the flow is parallel to the boundaries. The mesh dimensions can be adapted to the curved walls, but for simplicity it was preferred to use the same mesh and stepwise to approximate the curved walls. The resulting ragged boundaries cause fluctuations which vary depending on the number of points.

The resulting set of equations are arranged in a matrix and solved on a computer. From the ratio of pressure and velocity at the entrance the input impedance is calculated; mean values are taken over the cross-sections at distances $0, h$ and $2 h$ from the entrance. The velocity at the input, 0 , is proportional to the local gradient, which is obtained by applying 
Eq. (25) to the pressure values at positions 0,1 and 2 , giving $p_{0}^{\prime}=\left(-3 p_{0}+4 p_{1}-p_{2}\right) / 2 h$. The number of points was increased up to the capacity limit of the computer, about 40000 points. The results were plotted against the reciprocal number of points. The irregular fluctuations due to the rectangle-filled circular cross-section were leveled; the error due to the limited number of points was minimized by extrapolation to zero, i.e. to an infinite number of points.

The effect of the bend was found by comparing two pipe combinations of the same length, one without and one with a bend. The frequency was varied until either the input pressure or the input velocity was zero, corresponding to zero or infinite impedance. Although the frequency of the straight pipe can also be determined analytically, the FDM results were preferred, since the limited number of grid points resulted in small systematic errors; they were assumed to be the same for straight and curved pipes. The frequency corrections were recalculated in length corrections with Eq. (5) and referred to the tube radius $a$. At higher frequencies the wavelength is no longer large compared to the grid size, reducing the accuracy. The accuracy of the final result is estimated to be $0.02 a$ at the lowest, increasing up to $0.1 a$ at the highest frequencies.

\section{RESULTS}

Consider a duct combination with the same dimensions as the duct drawn in Fig. 1; $l_{\mathrm{u}} / a=3, l_{\mathrm{b}} / a=4 \pi / 3, l_{\mathrm{d}} / a=2$, with an ideal open end at the output: $p(s=L, r, \theta)=0$. Fig. 3 shows the impedance at the input end of the duct as a function of the frequency, and the corresponding length correction, together with the length corrections calculated with Eq. (19) and the FDM. This plot clearly points out the strong dependence with frequency of the mistuning induced by the bending. As reported in earlier studies, ${ }^{2 / 6}$ the length correction at low frequencies is negative. When increasing the frequency, it becomes smaller in absolute value (some oscillations can be observed, they are discussed

in the following), to reach zero at a frequency close to $f_{\mathrm{c}} / 2$. Then its sign changes and 
it increases, until it reaches, near the cutoff, a value that is generally comparable to its value at zero frequency, with opposite sign. Furthermore, in this example, the length correction is far from negligible, varying in absolute value from 0 to a quarter of the radius $a$.

It may be noted that the solid curve is not drawn until the cutoff frequency. This is because the procedure of calculation of the length correction [Eqs. (9) and (10)] fails as soon as the higher order modes, although still evanescent, can no longer be considered as a small perturbation.

When varying the curvature of the bent portion, while keeping constant the lengths $l_{\mathrm{u}}, l_{\mathrm{b}}$ and $l_{\mathrm{d}}$, the evolution with frequency of length correction is roughly the same, with mainly the overall amplitude of the curve changing: the larger the curvature, the larger the length correction, or, in other words, the stronger the mistuning (Fig. 4). At a given frequency, the length correction increases roughly as the square of the curvature $\kappa$ (Fig. 5).

Besides, the curves in Fig. 4 display particular behaviors that are almost independent of the curvature:

A. the length correction passes through a minimum in absolute value - nearly zero - at the vicinity $f=0.2 f_{\mathrm{c}}$,

B. the sign of the length correction changes at a frequency close to $f_{\mathrm{c}} / 2$.

\section{A. Minima of the length correction}

Following the low frequency description made by Nederveen ${ }^{213}$ and Keefe and Benade ${ }^{4}$ (cf. Sec. II.B), one may expect the effect of curvature to vanish, or at least be lowered, when the position of the bend in a duct coincides with a node of the longitudinal velocity field. Indeed, if one supposes that the velocity node is located at the center of the bend in the 


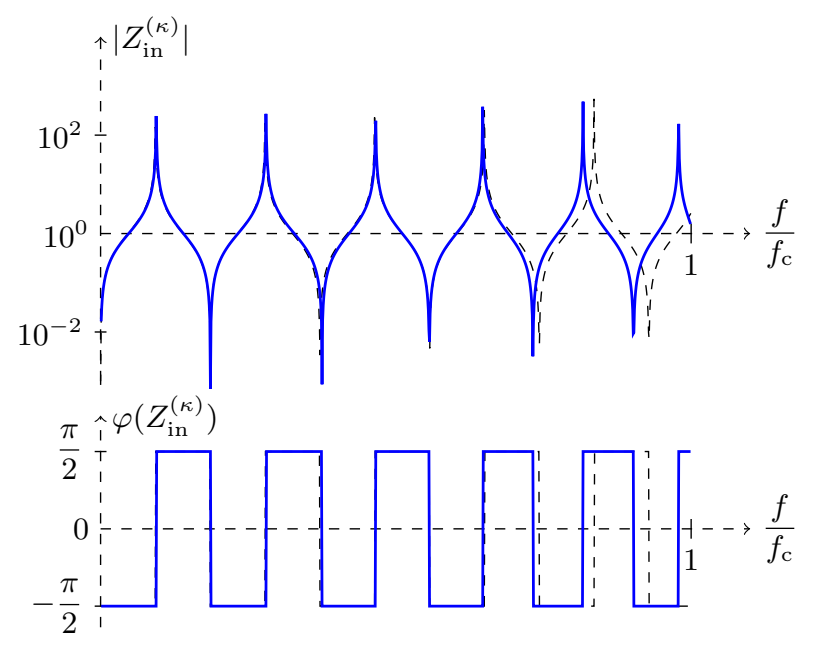

(a) Input impedance

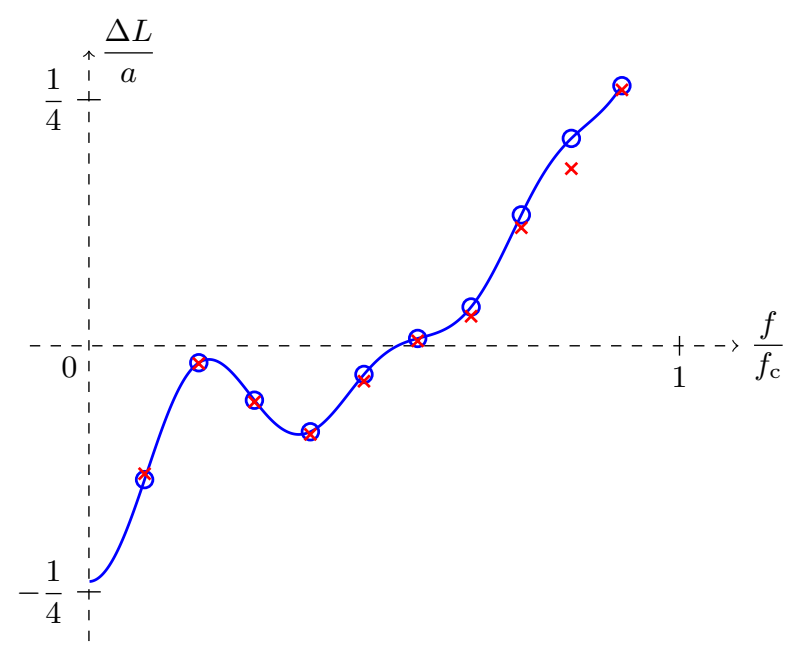

(b) Length correction

FIG. 3. (a) Input impedance (modulus and phase) of the duct shown in Fig. 11, with $l_{\mathrm{u}} / a=3$, $l_{\mathrm{b}} / a=4 \pi / 3, l_{\mathrm{d}} / a=2$, and the "open" boundary condition $p=0$ at the output end. The results, given for a curvature $\kappa=0.5$ of the bend axis and computed with 12 modes taken into account (solid curve), are compared with the input impedance of a straight duct $(\kappa=0$, dashed) with the same axis length $L / a$. (b) Length correction, in tube radius unit, for the same duct. Solid curve: length correction as defined by Eqs. (9) 10), 'o': solutions of the dispersion relation (19) with a "closed" or "open" condition at the input end, ' $\times$ ': finite differences method. 


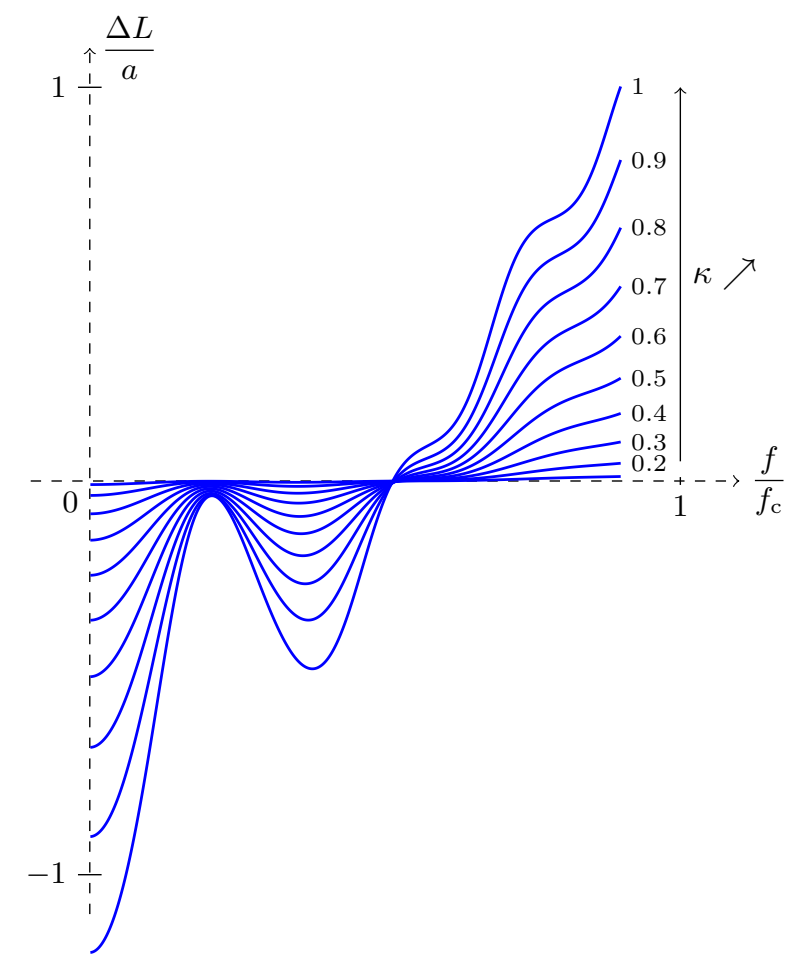

FIG. 4. Influence of the curvature on the length correction, as defined by Eqs. (9-10). The value of $\kappa$ is increased from 0.1 (the curve, not labeled, is close the abscissa axis) to 1 , in steps of 0.1 .



FIG. 5. Influence of the curvature on the length correction, as defined by Eqs. 99 10p: $\Delta L$ increases as, roughly, the square of $\kappa$, at a given frequency. 
example of Fig. 3, it follows that $\lambda / 4=l_{\mathrm{b}} / 2+l_{\mathrm{d}}$, where $\lambda$ is the wavelength. Thus,

$$
\frac{f}{f_{\mathrm{c}}}=\frac{\pi a}{\left(l_{\mathrm{b}}+2 l_{\mathrm{d}}\right) \gamma_{10}} \simeq 0.2084
$$

If one varies the distance $l_{\mathrm{d}}$ from the output to the bend while keeping constant the curvature $\kappa$, the axis length of the bend $l_{\mathrm{b}}$, and the overall length of the duct $L$, one observes a displacement of this local minimum of $|\Delta L|$ according to Eq. (28). The length correction does not exactly reach zero because at this frequency, the wavelength $(\lambda / a \simeq 16.4)$ is not large compared with the length of the bend $\left(l_{\mathrm{b}} / a \simeq 4.2\right)$. Thus, the velocity field is not zero or negligible in the whole bend.

Besides, one can deduce that the length correction for a long duct with a bent portion will exhibit a large number of local minima (Fig. 6). In this case, the first minima appear at long wavelengths, compared with the bend size, therefore the length correction $|\Delta L|$ is close to zero at these frequencies.

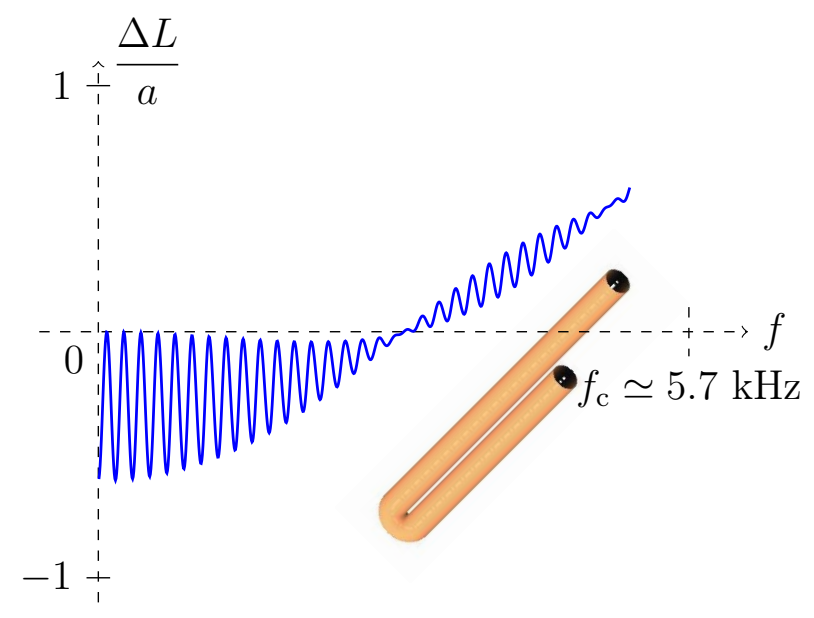

FIG. 6. Length correction, in tube radius unit, for a duct with $2 a=0.035 \mathrm{~m}, \kappa=0.78$, $l_{\mathrm{u}}=1.3 \mathrm{~m}, l_{\mathrm{b}}=\pi a / \kappa, l_{\mathrm{d}}=1 \mathrm{~m}$ (the duct is somewhat similar to the air column of a contrabass clarinet), and an "ideal open end" condition $p=0$ at the output end $(s=L)$. 


\section{B. Frequency of the sign change}

For frequencies below, and not too close to, the cutoff frequency $f_{\mathrm{c}}$, the properties of the bent segment, notably the way the length correction varies with frequency, are mainly related to the characteristics of the first bend mode $\phi_{0}^{(\kappa)}$. As mentioned before (cf. Sec. II.C), it is possible to approximate this mode and its associated propagation constant $k_{0}^{(\kappa)}$, by computing the eigenmodes of the truncated matrix $\mathcal{M}$ (Eq. 17). The propagation constant $k_{0}^{(\kappa)}$ is an increasing function of the frequency, that is lower than the propagation constant $k_{0}^{(0)}=k$ of the plane wave in a cylinder at low frequencies and higher near the cutoff (Fig. 7). A similar observation was made by Cummings ${ }^{18}$ in bends with rectangular cross-section, and borne out by El-Raheb. ${ }^{19}$ Therefore, if one assumes that the effect of the curvature at low frequencies is, roughly, to replace the plane wave propagating with a propagation constant $k_{0}^{(0)}=k$ by a quasi-plane wave propagating with the propagation constant $k_{0}^{(\kappa)}$, this explains qualitatively the variations of $\Delta L$ and the sign change.



FIG. 7. Evolution with frequency of the propagation constant $k_{0}^{(\kappa)}$ of the first bend mode in a bend with curvature $\kappa=0.5$, computed with 12 modes taken into account $\left(k_{\mathrm{c}}=2 \pi f_{\mathrm{c}} / c_{0}\right)$.

If one takes into account only two modes in the multimodal formulation - the plane wave mode $\phi_{0}^{(0)}$ and the first evanescent mode $\phi_{1}^{(0)}$ - an approximation of $k_{0}^{(\kappa)}$ can be analytically calculated:

$$
\begin{aligned}
k_{0}^{(\kappa)}= & {\left[k^{2}\left(1+\beta^{2} \kappa^{2}\right)+\ldots\right.} \\
& \left.\frac{1}{2}\left(\sqrt{k_{\mathrm{c}}^{4}-8 \beta^{2} \kappa^{2} k^{2}\left(k_{\mathrm{c}}^{2}-2 k^{2}\right)}-k_{\mathrm{c}}^{2}\right)\right]^{1 / 2}
\end{aligned}
$$


where

$$
\beta=\frac{1}{\gamma_{10}^{2} \sqrt{\frac{1}{2}\left(1-\frac{1}{\gamma_{10}^{2}}\right)}} \simeq 0.5
$$

It differs from the "converged" value, computed with 12 modes (Fig. 7), by less than $0.3 \%$. The frequency at which $k_{0}^{(\kappa)}=k$ is

$$
f_{\mathrm{s}}=\frac{f_{\mathrm{c}}}{2}\left[1-\left(\frac{\beta \kappa}{2}\right)^{2}\right] .
$$

It is weakly dependent of the curvature: the relative shift from $k_{\mathrm{c}} / 2$ varies between 0 and $\sim 6 \%$.

Of course, from this result, the rigorous calculation that would lead to the properties of the length correction itself is not straightforward, but this gives basic elements to better understand how a bend can modify the propagation and resonances in a duct.

Another remark can be made from the results on $k_{0}^{(\kappa)}$ : the inertance correction 12 was first deduced by Nederveen ${ }^{2}$ from a simplified formulation of the conservation equations in the long wavelength limit [Eq. (11)]. If, in a rough approximation, a low frequency wave in a bend is assumed to propagate with a propagation constant $k_{0}^{(\kappa)}$, then the coefficient

$\alpha$ should be related to the zero frequency limit of $\left(k_{0}^{(\kappa)} / k\right)^{2}$. Indeed, the relative difference between $\alpha$ and this limit remains under $5 \%$ until $\kappa \simeq 0.96$ (Fig. 8).

\section{EXPERIMENTAL VERIFICATION OF THE FREQUENCY SHIFT DUE TO THE CURVATURE}

To test the results, a duct was made in the workshop with a constant diameter $2 a=35 \mathrm{~mm}$ and a toroidal bend with a $\kappa$ of $7 / 9$, somewhat similar to the bend in a contrabass clarinet (Fig. 9). Its input impedance was measured using a set-up developed jointly by LAUM (Laboratory of Acoustics, Université du Maine, Le Mans, France) and CTTM (Center for Technology Transfer, Le Mans, France) ${ }^{20}$ It consists in placing at the input 


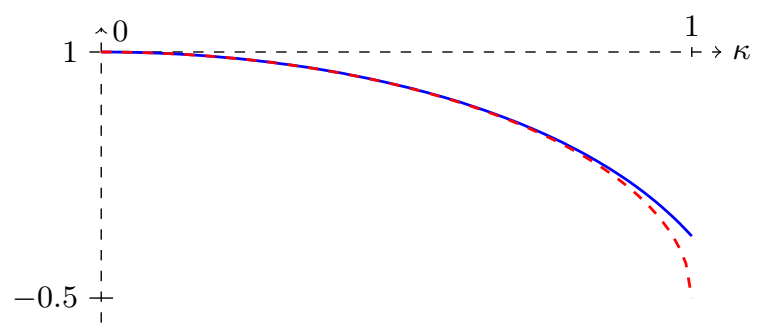

FIG. 8. Solid curve: zero frequency limit of $\left(k_{0}^{(\kappa)} / k\right)^{2}$, as approximated by its value at $f / f_{\mathrm{c}} \simeq 5 \cdot 10^{-6}$, computed with 12 modes taken into account, as a function of the curvature. Dashed curve: inertance correction $\alpha$ (Eq. 12).

of the measured instrument a piezo-electric source with a small closed cavity at its back. While a first microphone measures the pressure at the input of the instrument, a second microphone measures the pressure in the back cavity. Assuming the latter to be proportional to the volume velocity of the source, the input impedance of the measured object is then calculated from the transfer function between the two microphones, assuming that the motion of the piezo-electric transducer results in equal but out of phase volume velocity on both sides of the transducer. The reader may refer to Ref ${ }^{211}$ for details on this set-up.

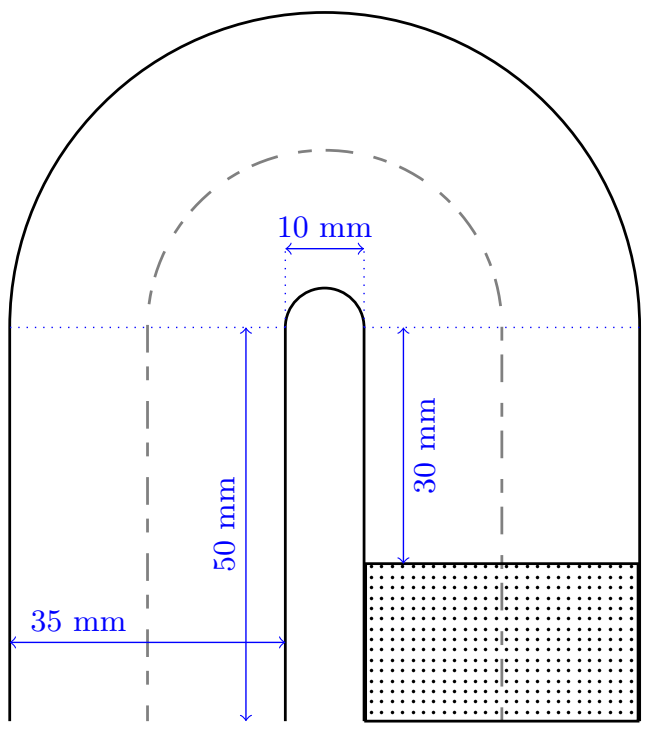

FIG. 9. Dimensions of the duct designed for the experiments. The axis curvature of the bend is $\kappa=7 / 9$ and the duct is closed at the output. 
The frequency range of interest, from zero to the first cutoff $f_{\mathrm{c}} \simeq 5.76 \mathrm{kHz}$, is decomposed in four overlapping intervals, in order to maximize the signal-to-noise ratio (SNR) without saturation of the measured signal. For each interval, a $1 \mathrm{~s}$ long logarithmic chirp is used as input signal, leading to a frequency resolution of $1 \mathrm{~Hz}$. The temperature in the tube is determined from a prior measurement of the input impedance of a closed cylindrical tube of known dimensions. For each tube, the acquisition is repeated five times and averaged. The calibration of the impedance sensor is described in the paper by Macaluso and Dalmont.21 The residual error is evaluated by calculating the difference between the measured input impedance of the straight tube with the theoretical value. Then the difference is used (except at its "singularities," that is, at the resonances of the straight tube) as a correction to the measured input impedance of the curved tube.

The measured input impedance is shown in Fig. 10(a). It is compared with the theoretical input impedance, computed as described earlier in the paper. To take into account the visco-thermal losses at the walls in the multimodal formulation, the purely real or purely imaginary propagation constants of the modes $\phi_{n}^{(0)}$ are replaced by complex propagation factors $\frac{1322}{122}$ Note, however, that this way of introducing the losses is not fully rigorous, since it assumes that the wall on which the boundary layer lies is locally plane. Therefore, the effect of the curvature of the boundary layer is not taken into account. Further investigations will be made on this particular aspect. In Eq. (9) the wavenumber $k$ is replaced by a complex wave number, as classically done. 23

Fig. 10(b) shows the comparison of the measured length correction with the theoretical and numerical results from the multimodal and finite difference methods. The agreement is good over the whole frequency range. The shift of about 0.05 between the two curves can be explained by a possible temperature difference between the two measured tubes and the uncertainty in the temperature evaluation from the input impedance of the straight tube. 


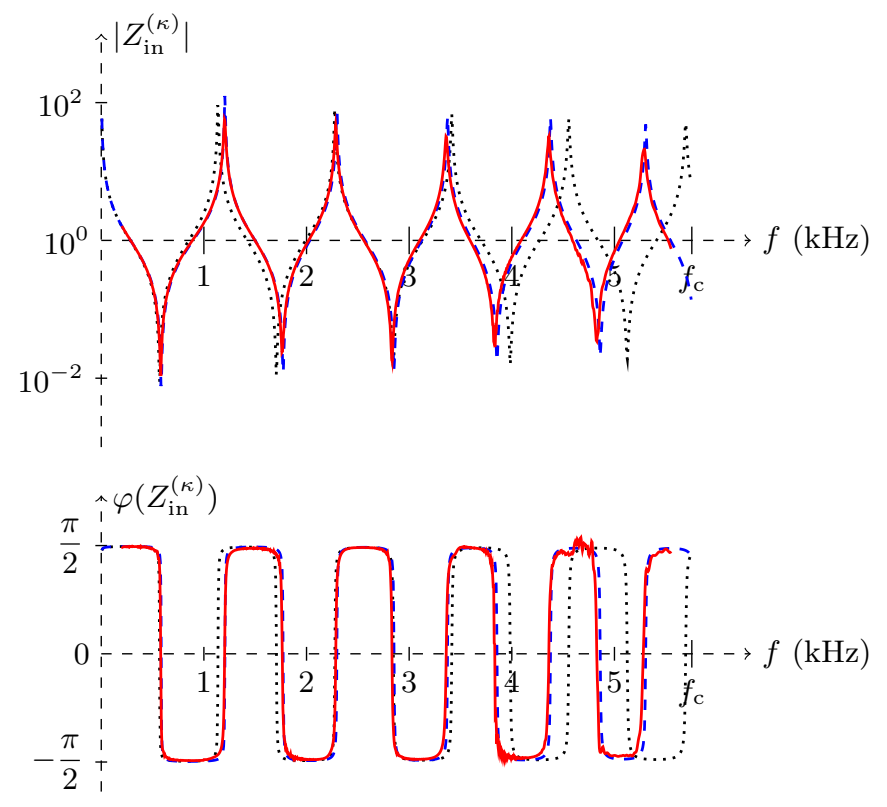

(a) Input impedance



(b) Length correction

FIG. 10. (a) Input impedance (modulus and phase) and (b) length correction for the duct shown in Fig. 9. Solid: experimental results. Dashed: theoretical results (the theoretical and experimental impedance curves are almost superimposed). Dotted: theoretical input impedance of a straight duct having the same radius and length. 'o': Solutions of Eq. (19) with a "closed" or "open" condition at the input end. ' $x$ ': Finite differences method. 


\section{CONCLUSION}

The effects of bending a portion of a duct have been investigated theoretically and experimentally. Results show that the frequency shift induced by the curvature is not always small and, moreover, is strongly frequency dependent. It notably exhibits a sign change, so that the first resonance frequencies in a bent tube are shifted towards higher values, while they are shifted towards lower values near the cutoff. It has been shown that the magnitude of the frequency shift varies, roughly, as the square of the curvature, and that the frequency of the sign change is weakly dependent on the curvature. In this paper, only lossless or weakly lossy cases have been considered, with an approximate description of the visco-thermal losses at walls. Earlier studies have shown, however, the significant influence of the curvature on the wave propagation in a lined bend, notably the effect of localized wall $\operatorname{losses}^{8}$. Future studies of this matter should then involve a more comprehensive study of the lossy propagation in a bend.

\section{Acknowledgments}

The authors wish to thank Joël Gilbert for fruitful discussions.

\section{References}

1 J. W. S. Rayleigh, Theory of Sound, volume 2, section 263 (McMillan and Company, London) (1878).

2 C. J. Nederveen, Acoustical aspects of woodwind instruments, 2nd edition, pp. 60-62 (Northern Illinois University Press, Dekalb, IL) (1998).

3 C. J. Nederveen, "Influence of a toroidal bend on wind instrument tuning", J. Acoust. Soc. Am. 104, 1616-1626 (1998).

4 D. H. Keefe and A. H. Benade, "Wave propagation in strongly curved ducts", J. Acoust. Soc. Am. 74, 320-332 (1983). 
5 G. S. Brindley, "Speed of sound in bent tubes and the design of wind instruments", Nature 246, 479-480 (1973).

6 J. W. Coltman, "Compensating for miter bends in cylindrical tubing", J. Acoust. Soc. Am. 121, 2497-2498 (2007).

7 S. Félix and V. Pagneux, "Multimodal analysis of acoustic propagation in threedimensional bends", Wave Motion 36, 157-168 (2002).

8 S. Félix and V. Pagneux, "Sound attenuation in lined bends", J. Acoust. Soc. Am. 116, 1921-1931 (2004).

9 S. Félix and V. Pagneux, "Sound propagation in rigid bends: A multimodal approach", J. Acoust. Soc. Am. 110, 1329-1337 (2001).

10 S. Félix and V. Pagneux, "Ray-wave correspondence in bent waveguides", Wave Motion 41, 339-355 (2005).

11 C. K. W. Tam, "A study of sound transmission in curved duct bends by the Galerkin method", J. Sound Vib. 45, 91-104 (1976).

12 V. Pagneux, N. Amir, and J. Kergomard, "A study of wave propagation in varying crosssection waveguides by modal decomposition - part 1: Theory and validation", J. Acoust. Soc. Am. 100, 2034-2048 (1996).

13 N. Amir, V. Pagneux, and J. Kergomard, "A study of wave propagation in varying crosssection waveguides by modal decomposition - part II: Results", J. Acoust. Soc. Am. 101, $2504-2517$ (1997).

14 S. V. Patankar, Numerical heat transfer and fluid flow, p. 59 (Taylor and Francis, London) (1980).

15 C. J. Nederveen and J.-P. Dalmont, "Corrections to the plane-wave approximation in rapidly flaring horns", Acta Acustica united with Acustica 94, 461-473 (2008).

16 D. Zwillinger, Handbook of differential equations, p. 579 (Academic Press, New York) (1992).

17 M. Abramowitz and I. A. Stegun, Handbook of Mathematical Functions, p. 883 (Dover, New York) (1970). 
18 A. Cummings, "Sound transmission in curved duct bends", J. Sound Vib. 35, 451-477 (1974).

19 M. El-Raheb, "Acoustic propagation in rigid three-dimensional waveguides", J. Acoust. Soc. Am. 67, 1924-1930 (1980).

20 J.-P. Dalmont and J.-C. Le Roux, "A new impedance sensor for wind instruments (A)", J. Acoust. Soc. Am. 123, 3014 (2008).

21 C. A. Macaluso and J.-P. Dalmont, "Trumpet with near-perfect harmonicity: Design and acoustic results", J. Acoust. Soc. Am. 129, 404-414 (2011).

22 A.-M. Bruneau, M. Bruneau, P. Herzog, and J. Kergomard, "Boundary layer attenuation of higher order modes in waveguides", J. Sound Vib. 119, 15-27 (1987).

23 J.-P. Dalmont, K. Nederveen, and N. Joly, "Radiation impedance of tubes with different flanges: Nnumerical and experimental investigations", J. Sound Vib. 244, 505-534 (2011). 


\section{List of Figures}

FIG. 1 Duct with a bent portion. $s$ is the abscissa along the waveguide axis, measured from the input of the duct (top), and $L=l_{\mathrm{u}}+l_{\mathrm{b}}+l_{\mathrm{d}}$ is the total length of the duct axis. . . . . . . . . . . . . . . . . . . . . . 4

FIG. 2 Grid layout for the FDM solution in a bent segment connected to a straight segment. . . . . . . . . . . . . . . . . . . 12

FIG. 3 (a) Input impedance (modulus and phase) of the duct shown in Fig. 1], with $l_{\mathrm{u}} / a=3, l_{\mathrm{b}} / a=4 \pi / 3, l_{\mathrm{d}} / a=2$, and the "open" boundary condition $p=0$ at the output end. The results, given for a curvature $\kappa=0.5$ of the bend axis and computed with 12 modes taken into account (solid curve), are compared with the input impedance of a straight duct $(\kappa=0$, dashed) with the same axis length $L / a$. (b) Length correction, in tube radius unit, for the same duct. Solid curve: length correction as defined by Eqs. (9-10), 'o': solutions of the dispersion relation (19) with a "closed" or "open" condition at the input end, ' $\times$ ': finite differences method. . . . . . . . . . . . . . . . . . . . . 16

FIG. 4 Influence of the curvature on the length correction, as defined by Eqs. (9+ 10). The value of $\kappa$ is increased from 0.1 (the curve, not labeled, is close the abscissa axis) to 1 , in steps of 0.1. . . . . . . . . . . . . . . . . . 17

FIG. 5 Influence of the curvature on the length correction, as defined by Eqs. (9) 10):

$\Delta L$ increases as, roughly, the square of $\kappa$, at a given frequency. . . . . . . . 17

FIG. 6 Length correction, in tube radius unit, for a duct with $2 a=0.035 \mathrm{~m}, \kappa=0.78$, $l_{\mathrm{u}}=1.3 \mathrm{~m}, l_{\mathrm{b}}=\pi a / \kappa, l_{\mathrm{d}}=1 \mathrm{~m}$ (the duct is somewhat similar to the air column of a contrabass clarinet), and an "ideal open end" condition $p=0$ at the output end $(s=L)$. . . . . . . . . . . . . . . . . . . . . . . . . . . . . 18

FIG. 7 Evolution with frequency of the propagation constant $k_{0}^{(\kappa)}$ of the first bend mode in a bend with curvature $\kappa=0.5$, computed with 12 modes taken into account $\left(k_{\mathrm{c}}=2 \pi f_{\mathrm{c}} / c_{0}\right) . \ldots \ldots \ldots \ldots \ldots \ldots$ 
FIG. 8 Solid curve: zero frequency limit of $\left(k_{0}^{(\kappa)} / k\right)^{2}$, as approximated by its value at $f / f_{\mathrm{c}} \simeq 5.10^{-6}$, computed with 12 modes taken into account, as a function of the curvature. Dashed curve: inertance correction $\alpha$ (Eq. [12). . . . . . . . 21

FIG. 9 Dimensions of the duct designed for the experiments. The axis curvature of the bend is $\kappa=7 / 9$ and the duct is closed at the output. . . . . . . . . . 21

FIG. 10 (a) Input impedance (modulus and phase) and (b) length correction for the duct shown in Fig. 9| Solid: experimental results. Dashed: theoretical results (the theoretical and experimental impedance curves are almost superimposed). Dotted: theoretical input impedance of a straight duct having the same radius and length. 'o': Solutions of Eq. (19) with a "closed" or "open" condition at the input end. ' $x$ ': Finite differences method. . . . . . . . . . . 23 